\title{
Metabolic bone diseases: an overview
}

\author{
L. Sinigaglia \\ Division of Rheumatology, Gaetano Pini Institute, Milan, Italy
}

$\mathrm{O}$ ver the last decade, the field of metabolic bone diseases has been marked by enormous advances, yet it still poses some significant challenges in the rheumatologic clinical practice. Bone pain, be it related or unrelated to fragility fractures, can be the presenting symptom of a variety of diseases frequently mimicking rheumatic conditions. Therefore bone health is a very relevant target in the rheumatologic practice that can only be achieved through a dedicated diagnostic and therapeutic approach based on a very thorough assessment of the clinical picture of each patient.

In this issue of Reumatismo we offer an update on a number of clinical conditions that are particularly relevant to rheumatologists in their clinical practice. The following articles aim to stir intellectual curiosity and draw attention to a more systematic evaluation of the role that bones play in the pathophysiology and outcomes of rheumatic diseases in keeping with the concept that rheumatology is the science covering all non-surgical diseases potentially affecting the musculoskeletal system as a whole.

The first article is devoted to the new therapies for osteoporosis which have opened up a new era offering new treatment options aimed at specific targets. These emerging therapies, be they based on biologic agents or metabolic pathway inhibitors, are definitely required in this field, since these fragile patients still have unmet needs in terms of further reduction of fracture risk, long-term side effects and common clinical problems resulting from poor compliance to current therapeutic regimens (1). The innovative drugs appearing on the horizon as a result of translational research efforts will be able to influence the osteoclast activity and significantly trigger bone neoformation, thus offering new potential opportunities to counteract skeletal fragility and ensuing fractures.

Three articles are devoted to specific models of osteoporosis which can be particularly intriguing for rheumatologists. Skeletal fragility in rheumatic diseases is not only confined to rheumatoid arthritis (2), but it may also be an emerging problem in a variety of other rheumatic conditions $(3,4)$ and has in general a multifactorial etiology related to chronic inflammation, loss of mobility and drugs used to treat the basic condition. Fragility fractures in rheumatic diseases represent an additional clinical challenge that directly affect the global health of the patients and induce a supplementary burden on their quality of life. The article on osteoporosis in rheumatic diseases focuses on the epidemiology (5) and the main underlying pathophysiologic mechanisms not only of rheumatoid arthritis but also of other rheumatic conditions for which recent data has highlighted the importance of skeletal involvement. Osteoporosis in male patients represents yet another exciting clinical challenge, since it most often appears when a fragility fracture occurs. Bone loss and fragility fractures in men, having potentially different underlying mechanisms from those of post-menopausal osteoporosis, have now been recognized as a serious and often equally neglected medical condition (6). New screening and diagnostic strategies as well as therapeutic options are listed in another article devoted to this topic. Lastly, epidemiology, pathophysiol-
Corresponding author: Luigi Sinigaglia Division of Rheumatology Gaetano Pini Institute, Milan, Italy E-mail: sinigaglia@gpini.it 
ogy and management of glucocorticoidinduced osteoporosis (7) are addressed in another article which reports the most recent insights in the mechanisms of skeletal damage induced by these drugs and the recent reviews of the main international guidelines on prevention and treatment of this common iatrogenic disease addressed by rheumatologists.

The role of Vitamin D in rheumatic diseases has been extensively studied in these last few years both for the important role of this hormone for bone health and its wellrecognized extra-skeletal effects. Recent data collected in Italy confirmed a high prevalence of hypovitamosis D in a large cohort of patients with rheumatoid arthritis (8) even after supplementation with standard doses of this hormone (9). More data showed that low levels of vitamin D are common also in other rheumatic diseases and hypovitaminosis D may have a role not only in the skeletal health of these patients, but also in the progression of the disease and even in the occurrence of some rheumatic conditions.

The only article on a single disease in this issue deals with Paget's bone disease, owing to recent advances in epidemiology, clinical diagnosis (10), pathogenesis and in the understanding of genetic and environmental factors involved in this common bone disease. This article also includes an extensive review of therapeutic options in an attempt to provide an overview of the latest clinical approach to this disease.

Finally, a whole article is devoted to a clinical overview of the bone marrow edema which represents a final common pathway of a variety of frequent clinical conditions with different etiologies. This condition is often associated with bone pain and has probably a different meaning (and a different substrate) in many rheumatic conditions, such as osteoarthritis, rheumatoid arthritis, spondyloarthritis and bone vascular syndromes, therefore its detection, classification and possibly treatment (11-13) may have important consequences in the daily rheumatologic practice.

The ultimate goal of this issue has been to collect in a single volume the latest knowl- edge about some crucial aspects of bone metabolic disorders which are particularly relevant for their rheumatologic implications and we hope and wish that this effort will be of value to all rheumatologists in their clinical practice.

\section{REFERENCES}

1. Rossini M, Bianchi G, Di Munno O, Giannini S, Minisola S, Sinigaglia L, et al. Determinants of adherence to osteoporosis treatment in clinical practice. Osteoporos Int. 2006; 17: 914-21.

2. Sinigaglia L, Nervetti A, Mela Q, Bianchi G, Del Puente A, Di Munno O, et al. A multicenter cross sectional study on bone mineral density in Rheumatoid arthritis. Italian Study Group on bone mass in Rheumatoid Arthritis. J Rheumatol. 2000; 27: 2582-9.

3. Sinigaglia L, Varenna M, Binelli L, Zucchi F, Ghiringhella D, Gallazzi M, et al. Determinants of bone mass in systemic lupus erythematosus: a cross sectional study on premenopausal women. J Rheumatol. 1999; 26 : 1280-4.

4. Sinigaglia L, Varenna M, Casari S. Bone involvement in osteoarthritis. Sem Arthritis Rheum. 2005; 34: 44-6.

5. Sinigaglia L, Varenna M, Girasole G, Bianchi G. Epidemiology of osteoporosis in rheumatic diseases. Rheum Dis Clin N Am. 2006; 32: 631-58.

6. Varenna M, Sinigaglia L, Adami S, Giannini S, Isaia GC, Maggi S, et al. Association of quantitative heel ultrasound with history of osteoporotic fractures in elderly men: the ESOPO study. Osteoporos Int. 2005; 16: 1749-54.

7. Sinigaglia L, Mazzocchi D, Varenna M. Bone involvement in exogenous hypercortisolism. J Endocrinol Invest. 2008; 31: 364-70.

8. Rossini M, Maddali Bongi S, La Montagna G, Minisola G, Malavolta N, Bernini L, et al. Vitamin D deficiency in rheumatoid arthritis: prevalence, determinants and associations with disease activity and disability. Arthritis Res Ther. 2010; 12: R216.

9. Varenna M, Manara M, Cantatore FP, Del Puente A, Di Munno O, Malavolta N, et al. Determinants and effects of vitamin D supplementation on serum 25-hydroxy-vitamin D levels patients with Rheumatoid Arthritis. Clin Exper Rheumatol. 2012; 30: 714-19.

10. Varenna M, Zucchi F, Galli L, Manara M, De Marco G, Sinigaglia L. Demographic and clinical features related to a symptomatic onset of Paget's disease of bone. J Rheumatol. 2010; 37: 155-60. 
11. Varenna M, Zucchi F, Ghiringhelli D, Binelli L, Bevilacqua M, Bettica P, et al. Intravenous clodronate in the treatment of reflex sympathetic dystrophy syndrome. A randomized, double blind, placebo controlled study. J Rheumatol. 2000; 27: 1477-83.

12. Varenna M, Zucchi F, Binelli L, Failoni S, Gallazzi M, Sinigaglia L. Intravenous pami- dronate in the treatment of transient osteoporosis of the hip. Bone 2002; 31: 96-101.

13. Varenna M, Adami S, Rossini M, Gatti D, Idolazzi L, Zucchi F, et al. Treatment of complex regional pain syndrome type I with Neridronate: a randomized, double blind, placebo-controlled study. Rheumatology (Oxford). 2013; 52: 534-42. 